\title{
Pyrexia and a rash
}

\author{
Renee Hoenderkamp, Kirun Gunganah and Tahseen A Chowdhury
}

\section{Case presentation}

A 38-year-old Malaysian female was admitted with a five-week history of intermittent fevers, myalgia, arthralgia, sore throat and rash. Fevers occurred mostly in the evening, when the rash would also be worst. She denied symptoms of cough, dysuria, gastrointestinal symptoms or any other symptoms suggestive of an infective focus. She had no weight loss, foreign travel, significant family or sexual history. She had a past history of type 2 diabetes and hypertension, well controlled on oral medication.

On examination she was systemically well, blood pressure 102/67 mmHg, pulse 100 regular, but had frequent temperature spikes of up to $40^{\circ} \mathrm{C}$, particularly during the evenings. During the temperature spikes she became flushed and developed an erythematous, confluent rash mainly on the thighs and back. There was no lymphadenopathy or hepatosplenomegaly, and no sign of diabetic complications (including foot ulceration). Baseline investigations on admission are shown in Table 1.

\section{What is the differential diagnosis and most likely diagnosis?}

The differential diagnosis of pyrexia of uncertain origin (PUO) is myriad. In this patient, a viral aetiology (particularly H1N1 influenza) was thought to be the most likely diagnosis. While occult bacterial infection needed to be excluded, it was felt unlikely as the patient looked relatively well in between episodes of pyrexia. Other possibilities considered included tuberculosis, haematological malignancy, atypical infections or autoimmune/inflammatory conditions. The patient had no risk factors for HIV infection, and tropical infection was deemed unlikely due to her lack of recent travel abroad.

\section{What is the initial management?}

She was treated with antipyretics, intravenous fluids and oseltamivir was initiated following throat and nasal swabs. Antibiotics were commenced by the admitting team (co-amoxiclav and clarithromycin). She was re-examined frequently for any new clinical findings, and a full infection screen undertaken, including blood and urine cultures, viral titres, atypical infec-

Renee Hoenderkamp, foundation year 1 trainee; Kirun Gunganah, specialist trainee in diabetes and endocrinology; Tahseen A

Chowdhury, consultant in diabetes

Department of Diabetes and Metabolism, The Royal London Hospital tion screen, auto-antibodies. A computed tomography (CT) scan was undertaken to exclude lymphoma.

\section{Case progression}

Despite intravenous antibiotics, and oseltamivir, her fever spikes persisted. She developed a macular papular rash following

\section{Table 1. Baseline investigations.}

\begin{tabular}{|c|c|}
\hline Haemoglobin & $13.1 \mathrm{~g} / \mathrm{dl}(11.5-15.1)$ \\
\hline Platelets & $257 \times 10^{9} / 1(150-400)$ \\
\hline White cell count & $19.0 \times 10^{9} / /(4-11)$ \\
\hline Neutrophils & $17.7 \times 10^{9} / 1(3-10)$ \\
\hline Sodium & $134 \mathrm{mmol} / \mathrm{l}(136-146)$ \\
\hline Potassium & $4.0 \mathrm{mmol} / \mathrm{l}(3.5-5.1)$ \\
\hline Urea & $2.6 \mathrm{mmol} / \mathrm{l}(2.5-6.4)$ \\
\hline Creatinine & $49 \mu \mathrm{mol} / \mathrm{l}(62-106)$ \\
\hline Bilirubin & $13 \mathrm{IU} / \mathrm{I}(1-17)$ \\
\hline Alanine transaminase & $49 \mathrm{IU} / \mathrm{I}(7-35)$ \\
\hline Alkaline phosphatase & 174 IU/I (35-104) \\
\hline C-reactive protein & $423 \mathrm{mg} / \mathrm{l}(<5)$ \\
\hline Chest radiograph & $\begin{array}{l}\text { Normal. No consolidation, no } \\
\text { lymphadenopathy }\end{array}$ \\
\hline Urinalysis & Normal \\
\hline Electrocardiogram & Sinus tachycardia \\
\hline
\end{tabular}

Table 2. Yamaguchi criteria for diagnosis of adult onset Still's disease.

Diagnosis made on five criteria (with a minimum two major).

Major criteria (two points)

- $\quad$ Arthralgia $>2$ weeks

- $\quad$ Fever $>39^{\circ}$ intermittent $>1$ week

- Typical rash

- White cell count $>100,000$ ( $80 \%$ leucocytes)

Minor criteria (one point)

- $\quad$ Sore throat

- Lymphadenopathy $+/$ - splenomegaly

- Abnormal liver function tests

- Negative antinuclear antibody and rheumatoid factor 


\section{Key learning points}

- Pyrexia of uncertain origin (PUO) can present a significant diagnostic challenge.

- Adult onset Still's disease (AOSD) is an important cause of PUO, and is a diagnosis of exclusion.

- While a patient may fit diagnostic criteria for AOSD, other causes of rash and fever should be considered in those with atypical features.

- Mycoplasma infection may present with extra-pulmonary features and is a rare cause of urticarial vasculitis.

antibiotic administration, and co-amoxiclav was stopped, but Epstein-Barr serology was negative. CT chest/abdomen/pelvis were entirely normal, with no lymphadenopathy and no occult abscess. Multiple blood and urine cultures showed no growth. Liver function tests became progressively deranged, with rising transamines to a nadir of $500 \mathrm{IU} / \mathrm{l}$. Acute hepatitis screen was negative. Echocardiogram was unremarkable, and viral serology, throat swab, immunoglobulins, HIV and autoantibody screen returned negative.

By day eight, she continued to spike fevers. She complained of arthralgia of the wrists and fingers, but there was no sign of acute arthritis. Her rash became more apparent. She was noted to have a significantly elevated ferritin level at $14,487 \mathrm{mg} / \mathrm{dl}$. A presumed diagnosis of adult onset Still's disease (AOSD) was made and a rheumatological opinion sought. She was started on non-steroidal anti-inflammatories (NSAIDs), and her rash and fever rapidly improved. The rash was thought to be atypical of AOSD, and she thus underwent a skin biopsy and was discharged. At review two weeks later, her symptoms and inflammatory markers were improving.

\section{What is the likely diagnosis and how should this be managed?}

Following intensive investigation for infection and malignancy, a diagnosis of AOSD was deemed likely as she had many criteria fulfilling the diagnosis for the condition. In view of the atypical skin rash, a skin biopsy was done, and showed urticarial vasculitis. In addition, mycoplasma pneumonia antibody titre showed rise from 1:40 on admission to 1:160, suggesting acute mycoplasma infection, and a diagnosis of urticarial vasculitis following acute mycoplasma infection was made. As her symptoms were settling, she required no further treatment.

\section{Discussion}

Mycoplasma infection is common and usually presents with respiratory symptoms, classically an atypical pneumonia. Extra- pulmonary manifestations, such as rash and arthralgia, are uncommon but reported. The association of mycoplasma infection with urticarial vasculitis is rare, but has also been previously reported in the literature. Urticarial vasculitis tends to be a pathological diagnosis based on the finding of a leukocytoclastic vasculitis, and may be associated with low or normal complement levels.

This case highlights the difficulty of conclusive diagnosis of AOSD. AOSD is a rare systemic inflammatory disorder of unknown aetiology that may present with pyrexia of uncertain origin. ${ }^{1}$ Complications include joint destruction, pleuritis, pulmonary fibrosis, pericarditis, tamponade and myocarditis. ${ }^{2}$ The condition has a greater prevalence in women aged 16-25, although in case series from Asia, age of onset over 35 is seen. ${ }^{1}$ Aetiology is unknown but suggestions include a genetic component (HLA), altered cytokine production, or a reactive response to microbial triggers such as Epstein-Barr virus, parainfluenza and indeed mycoplasma pneumonia. ${ }^{1}$ The prognosis of AOSD is variable - some cases resolve after the first episode and never recur, but others lead to a longer term severe predominantly articular destructive course. NSAIDs or corticosteroids are first line, but disease modifying agents may be required.

In this patient, multiple investigations revealed no obvious source of infection, and with her clinical findings and raised ferritin, AOSD was deemed likely. Her findings fitted well with Yamaguchis's criteria, which are described as $93.5 \%$ sensitive. Nevertheless, an atypical rash and marked clinical response to NSAIDs suggested an alternative diagnosis. This case highlights the importance of keeping an open mind when diagnosing AOSD as a cause of PUO. ${ }^{4}$

\section{References}

1 Efthimiou P, Paik PK, Bielory L. Diagnosis and management of adult onset Still's disease. Ann Rheum Dis 2006;65:564-72.

2 Cagatay Y, Gul A, Cagatay A et al. Adult-onset Still's disease. Int J Clin Pract 2009;63:1050-5.

3 Lambotte P, Cacoub N, Costedoat G et al. High ferritin and low glycosylated ferritin may also be a marker of excessive macrophage activation. J Rheumatol 2003;30:1027-8

4 Perez C, Artola V. Adult still's disease associated with mycoplasma pneumoniae infection. Clin infect Dis 2001;32:E105-6.

Address for correspondence: Dr TA Chowdhury, Department of Diabetes and Metabolism, 7th Floor, John Harrison House, The Royal London Hospital, Whitechapel, London E1 1BB. Email: Tahseen.Chowdhury@bartsandthelondon.nhs.uk 